2002) outside their own field, and smaller texts providing a general overview for associated specialities are required. I am uncertain what audience this book has been written for but I believe it goes some way to meet the requirement of an update for the generalist.

For a multiple author book it is well edited and there is little repetition. It is relatively inexpensive and generally a very enjoyable read. It is well illustrated and well referenced. It should not be regarded as a reference text but rather as one that can easily be read from start to finish over a short period, and I believe it will update the reader with most of what is happening in paediatric urology. I do not think the specialist paediatric urologist will find much new here and the book will be of far more interest for the general paediatrician, paediatric surgeon, urologist, and trainees in those areas.

The book covers most of the major areas (but not all) of urinary tract pathology, including: prenatal diagnosis, vesicoureteral reflux, duplex systems, voiding dysfunction, neuropathic bladder, bladder exstrophy, stones, oncology, hypospadias, undescended testis, and urological emergencies.

One chapter deserves special mention: "Developmental perspectives of children with genitourinary anomalies". Conditions such as incontinence and genital abnormalities can be particularly worrying for children and adolescents, and the point is clearly made that "identity becomes entwined with anomaly" and as a result adolescents may become developmentally blocked. The importance of identifying abnormal development to prevent problems and to treat psychological conditions that have already developed, and the need for every paediatric urology service to have formal psychological and psychiatric support is clearly made. It is encouraging to see such a holistic approach to patient care.

The chapters on vesicoureteral reflux provide an excellent overview, and it is refreshing to find an American text adhering to evidence based principles on the role of surgery. Although the topic of urinary tract infection is alluded to in a number of chapters, it is disappointing that it was not covered in a separate chapter dealing with presentation, investigation, and treatment. I also think it would have been useful to include a general chapter on urinary tract obstruction rather than have it dealt with under prenatal diagnosis.

The chapters on hyospadias and bladder exstrophy are excellent, but for the general reader there is perhaps a little too much surgical detail. It is as if the authors were undecided about which audience they were aiming at. I also think it would have been useful if the chapter on exstrophy addressed long term management rather than concentrating on the initial closure of the bladder in infancy. This is of particular importance in the United Kingdom setting where the management of exstrophy is now limited to two national centres, and there is a need for referring paediatricians to know what will happen to their patients.

Despite these shortcomings, overall I think this book is a valuable contribution, and most paediatric and urology departments would benefit from having it on the shelves of their libraries.

P S J Malone

\section{Tuberous sclerosis complex: from basic science to clinical phenotypes}

Edited by Paolo Curatolo. Cambridge: Cambridge University Press, 2003. £55.00 (hardback), pp 301. ISBN 1898683395

Tuberous sclerosis has always had the capacity to confuse clinicians. Friedrich von Recklinghausen confused it with neurofibromatosis, when on 25 March 1862 he presented a case to the Obstetrical Society of Berlin. The case he described was of a young infant who had died soon after childbirth and who was discovered on postmortem examination to have multiple cardiac myomata in the ventricular walls and a "great number of scleroses" in the brain. It was a French physician, Desire Magloire Bourneville, a pupil of Charcot, who has won the plaudits for correctly appreciating that tuberous sclerosis was a separate disease. In 1879 he described the case of a 15 year old girl who died at the Salpetriere in Paris. She had suffered from epilepsy and severe learning difficulties for most of her life and was afflicted with a disfiguring vesicular-papular eruption on her face. An autopsy revealed that she had many hard sclerotic lesions in the cerebral cortex and white nodular lesions protruding into the lateral ventricles. Bourneville coined the term "tuberous sclerosis of the cerebral convolutions" for this unique pathology. The term tuberous sclerosis has stuck, although the French still patriotically talk of Bourneville's disease.

Part of the reason that tuberous sclerosis has confused us, and still does, is that there has been a relative lack of population based research of the disease. In 1908 Vogt proposed a triad of clinical features that he felt characterised the disease, namely seizures, mental handicap, and "adenoma sebaceum". No doubt the description accurately reflected his anecdotal clinical experience. It is still quoted in medical textbooks to this day. However, it grossly oversimplifies the disease. Epidemiological studies of the disease have revealed that more than half of those with tuberous sclerosis complex (TSC) will have a normal intellect and that complications related to the disease may occur in any organ of the body. The plea for more epidemiological research is not just an academic point. Patients may, and probably do, suffer if clinical decisions about their medical care are based on the results of hospital case series that are distorted by selection biases.

Professor Curatolo's book goes a long way to demystifying this complex disease. Occasionally the authors fall into the trap of uncritically quoting many of the aforementioned misleading case series. However, this is more than compensated for by some excellent chapters within the book on the neurological and neuropsychiatric complications of the disease. The piece on the cognitive and intellectual impairments of the disease is the most balanced and comprehensive summary of the subject to date. Bolton clearly points out that the majority of TSC individuals fall within the normal range of IQ, but also suggests that many of those with high intelligence may also have specific neuropsychological impairments. He also explores the question of what causes the intellectual impairments in TSC in some detail and concludes that it is likely to be related to not just the extent of the structural brain abnormality but also the age of onset and type of seizure disorder as well as the nature of the underlying genetic mutation.

Curatolo and Seri's chapter on seizures is also strong. Particularly interesting is the discussion of how epileptogenesis in TSC may be related to an impairment of GABA mediated neuro-inhibition. It is startling how well vigabatrin, a specific inhibitor of gamma amino butyric acid aminotransferase, is supposed to work in the treatment of infantile spasms in tuberous sclerosis. In Hancock and Osborne's Cochrane Review of the treatment of infantile spasms, they found that there was a $95 \%$ response rate in those individuals with TSC who were treated with vigabatrin. The acid test of this therapy, of course, will be to see whether the apparent remarkable success rate in stopping spasms is matched by an improved intellectual outcome in these patients later in life.

The genetics of TSC are fascinating, and Kwiatkowski and colleagues carefully and clearly take the reader through the complexities of the subject. The disease is caused by alterations in two genes, TSC 1 and TSC 2 , on chromosomes 9 and 16 respectively. Both are tumour suppressor genes and mutations in either can be responsible for all the complications seen in the disease. The gene products of the two genes, hamartin and tuberin, appear to act together in the regulation of cell growth by inhibiting a substance known as mTOR (target of rapamycin). In those individuals with the disease this inhibition is thought to be reduced and therefore there is the formation of the hamartomas that are characteristic of the disease. It is exciting that rapamycin, a clinically available compound, also selectively inhibits mTOR and therefore there is now the possibility that rapamycin may provide some benefit in reducing the clinical manifestations of TSC. Fittingly, Professor Curatolo's book finishes with the intriguing suggestion that at last we may be on the threshold of being able to do something to ameliorate this complex and potentially devastating disease.

F O'Callaghan

\section{CORRECTION}

doi: 10.1136/adc.2004.046805corr l

Toni S, Reali M F, Fasulo A, et al. The use of insulin pump improves the metabolic control in children and adolescents with type 1 diabetes Arch Dis Child 2004;89:796-7. The initial of Dr Festini was incorrectly published as an F. The correct initial is P. 